\title{
Carotid Body Chemoreceptor Regulation of Blood Glucose during Aerobic Exercise
}

\author{
Blair D. Johnson ${ }^{1}$, Ana B. Peinado², Jennifer L. Taylor ${ }^{1}$, Michael \\ J. Joyner, FACSM ${ }^{1} .{ }^{1}$ Mayo Clinic, Rochester, MN. ${ }^{2}$ Technical \\ University of Madrid, Madrid, Spain. \\ (No relationships reported)
}

Emerging evidence in humans indicates that the carotid body chemoreceptors (CB) contribute to blood glucose regulation and counter-regulatory responses to a hyperinsulinemic-hypoglycemic clamp; however, it is unclear if the CB contribute to blood glucose regulation during exercise in humans. PURPOSE To test the hypothesis that the CB contribute to blood glucose regulation during exercise. METHODS Six recreationally trained men completed two randomized exercise sessions at $65 \% \mathrm{VO}_{2}$ max for up to 2 hours. One session was performed with an IV infusion of dopamine ( $2 \mu \mathrm{g} / \mathrm{kg} / \mathrm{min}$ ) (DA) to blunt CB chemosensitivity, and the other with an IV infusion of saline (SAL). Blood was drawn for blood glucose and counter-regulatory hormones at baseline, resting infusion, and every 10 minutes during exercise. A hypoxic ventilatory response (HVR) test was performed 15 minutes following exercise, with a continuous infusion of DA or SAL. Subjects were classified as being responders (RS) if their HVR was lower during DA vs. SAL, and non-responders (NR) if their HVR during DA was equal to or greater than their HVR during SAL. RESULTS Three subjects were classified as RS as DA reduced their HVR from $2.14 \pm 0.2$ to $1.03 \pm 0.3 \mathrm{~L} / \mathrm{min} / \% \mathrm{~S}_{\mathrm{a}} \mathrm{O}_{2}$. Two RS were unable to complete the 2 hours of exercise during DA (mean $90 \pm 17.3$ minutes) while all RS completed the SAL session. In RS, blood glucose during DA had a greater change from baseline vs. SAL (condition main effect: $-14.0 \pm 3.3$ vs. $-5.0 \pm 3.1 \%, \mathrm{P}<0.03$ ) and cortisol during DA had a smaller change from baseline vs. SAL (condition main effect: $0.9 \pm 8.4$ vs. 23.2 $\pm 9.7 \%, \mathrm{P}<0.03$ ). There were no differences between DA and SAL for the percent change from baseline for growth hormone, glucagon, epinephrine, and norepinephrine in the RS group. Meanwhile, all three NR completed both DA and SAL sessions. In the NR group, there were no differences between DA and SAL for the percent change from baseline blood glucose, growth hormone, glucagon, cortisol, epinephrine, and norepinephrine. CONCLUSIONS DA administered at $2 \mu \mathrm{g} / \mathrm{kg} / \mathrm{min}$ was not effective in blunting CB chemosensitivity in all subjects. However, when the HVR is effectively blunted by DA, the CB appear to contribute to blood glucose regulation during prolonged exercise, possibly by diminishing the cortisol response to exercise. 